This is the accepted version of the article:

Chávez-Ángel, E.; Zarate, R.A.; Gomis-Bresco, J.; Alzina, F.; Sotomayor Torres, C.M.. Modification of Akhieser mechanism in Si nanomembranes and thermal conductivity dependence of the Q-factor of high frequency nanoresonators. Semiconductor Science and Technology, (2014). . : - . 10.1088/0268-1242/29/12/124010.

Available at:

https://dx.doi.org/10.1088/0268-1242/29/12/124010 


\title{
Modification of Akhieser mechanism in Si nanomembranes and thermal conductivity dependence of the Q-factor of high frequency nanoresonators
}

\author{
E. Chávez-Ángel ${ }^{1,2}$, R.A. Zarate ${ }^{3}$, J. Gomis-Bresco ${ }^{1}$, F. Alzina ${ }^{1}$ and \\ C.M. Sotomayor Torres ${ }^{1,4}$ \\ ${ }^{1}$ Catalan Institute of Nanoscience and Nanotechnology (ICN2), Campus UAB 08193 Bellaterra \\ (Barcelona), Spain. \\ ${ }^{2}$ Dept. of Physics, Universitat Autònoma de Barcelona, Campus UAB, 08193 Bellaterra (Barcelona), \\ Spain. \\ ${ }^{3}$ Dpto de Fisica, Universidad Católica del Norte, UCN, Av. Angamos 0610, Antofagasta, Chile. \\ ${ }^{4}$ Catalan Institution for Research and Advanced Studies (ICREA) 08010 Barcelona, Spain. \\ *E-mail corresponding author: emigdio.chavez@icn.cat
}

\begin{abstract}
We present and validate a reformulated Akhieser model that takes into account the reduction of the thermal conductivity due to the impact of boundary scattering on the thermal phonons lifetime. We consider silicon nanomembranes with mechanical mode frequencies in the GHz range as textbook examples of nanoresonators. The model successfully accounts for the measured shortening of the mechanical mode lifetime. Moreover, the thermal conductivity is extracted from the measured lifetime of the mechanical modes in the high-frequency regime, thereby demonstrating that the $Q$ factor can be used as an indication of the thermal conductivity and/or diffusivity of a mechanical resonator.
\end{abstract}

\section{Introduction}

Advances in nanofabrication methods have enabled the evolution from micro- to nanoelectromechanical systems (MEMS and NEMS) with a vast variety of applications ranging from semiconductor technology to fundamental science [1]. MEMS- and NEMS-based oscillators have been used as extremely sensitive mass [2], force [3], charge [4] and spin [5] sensors. Coupling of MEMS and NEMS to the electromagnetic field via optical cavities, known as optomechanic cavities [6] has led to structures which provide quantum-limited displacement sensitivity [7] and back-action between photons and phonons. These characteristics of optomechanical structures have enabled the amplification of localized mechanical vibrations and the ensuing demonstration of phonon lasing [8], as well as ground state cooling at the nanoscale [9]. 
As the operation frequency of mechanical resonators enters the GHz regime [10-16], the intrinsic damping mechanism arising from phonon-phonon scattering becomes dominant. It is precisely this mechanism that introduces a fundamental limit to the performance of the resonators, usually expressed by the " $Q \cdot f$ " product of the quality factor, $Q$, and the work frequency, $f$. Two different approaches, commonly referred as Akhieser [17] and LandauRumer [18] models, have been used to describe the phonon-phonon interaction damping processes. In the Akhieser model the mechanical wave is treated as a macroscopic strain field in the crystal, which produces a shift of the equilibrium distribution of thermal phonons. The displaced thermal phonon distribution returns to equilibrium via phonon-phonon scattering, resulting time-dependent entropy of the system that leads to the absorption of the mechanical wave. On the contrary, in the Landau-Rumer approach all phonons are described as colliding individual particles and the three-phonons scattering process is described by the Fermi golden rule, based on quantum mechanical perturbation theory and the anharmonicity of the crystal potential, therefore, it is important to consider the changes arising from the reduction in feature size since phonon-phonon scattering depends on the materials properties. In the nanoscale, the shortening of the phonon mean free path (phonon lifetime) due to diffusive scattering of phonons at the boundaries [19-22] reduces the thermal conductivity compared to the bulk value and, as a consequence, the intrinsic damping mechanism (phonon-phonon scattering) is modified.

Although theoretical models are well established, comparison with experimental data is hindered by the number and uncertainty of the parameters used, including the thermal phonon mean free path and the Grüneisen parameter [23]. Moreover, accurate lifetime measurements of high-frequency phonons are a formidable task and values remain unknown for most materials, with only few reports on GHz to THz phonons in Si and GaAs [24-28].

Here we report on the impact of size-dependent thermal properties on the intrinsic damping mechanism in a mechanical resonator taking a free-standing silicon nanomembrane [29] as the simplest example of a nanoscale mechanical resonator. Such membrane exhibits well-defined 
Lamb mechanical resonances with thickness-tuneable frequency in the $10 \mathrm{GHz}$ to $1 \mathrm{THz}$ range. The intrinsic mechanical mode absorption is calculated using the Akhieser damping model [17] modified to take into account the size effect on the thermal conductivity due to thermal phonons scattering at the boundaries. All the simulations are compared against the experimental measurements of references $[25,30]$.

Furthermore, we show that it is possible to extract the thermal conductivity from lifetime measurements, thus, introducing the prospect of using measurements of the $Q$-factor to estimate the thermal conductivity and or diffusivity of the resonator.

\section{Modelling of the mechanical mode decay}

The losses of the mechanical mode are divided into intrinsic and extrinsic contributions. While the first is related to the anharmonic decay, i.e., phonon-phonon scattering processes, the latter is due to scattering from defects, rough surfaces, interfaces, etc. The relative importance of these contributions varies with the temperature and the frequency of the mechanical mode.

For the intrinsic damping mechanism, we can distinguish two regimes of attenuation , namely Akhieser [17] and Landau-Rumer [18], which depend on the relationship between the phonon wavelength $\lambda$ and averaged mean free path of thermal phonons, $\Lambda_{m f p}$. Although the frequencies of the thermal phonons span several decades and their mean free path can vary over orders of magnitude [31,32], a single parameter $\Lambda_{m f p}$ is usually defined to encompass the whole spectrum of thermal phonons. Given this $\Lambda_{m f p}$ we can define an average thermal phonon lifetime as $\tau_{T H}=\Lambda_{m f p} / \bar{v}$, where $\bar{v}=\left(\left(1 / v_{l}^{3}+2 / v_{t}^{3}\right) / 3\right)^{-1 / 3}$ is the average Debye velocity and $v_{l(t)}$ is the longitudinal (transverse) velocity. $\tau_{T H}$ is linked to the thermal conductivity $\kappa$ through the expression [33]

$$
\tau_{T H} \approx \frac{3 \kappa}{C_{V} \bar{v}^{2}}
$$

where $C_{V}$ is the volumetric heat capacity. If the wavelength $\lambda$ of the acoustic wave is much larger than the typical averaged mean free path $\Lambda_{m f p}$ of the thermal phonons, i.e., $f \cdot \tau_{T H}<<1$ where $f$ is the frequency of the acoustic wave, we can assume that the acoustic wave interacts 
with the whole spectrum of thermal phonons. This is the range known as the Akhieser regime. On the other hand, if $\lambda$ is much less than $\Lambda_{m f p}$, i.e., $f \cdot \tau_{T H} \gg>1$, then we describe the phonon attenuation using the Landau-Rumer formalism and the phonon relaxation is assumed to be a three-phonon scattering process.

In the Akhieser regime the intrinsic phonon relaxation rate of a mode $s$ with angular frequency $\omega_{s}=2 \pi f_{s}$ can be expressed as $[17,24,34]$ :

$$
\tau_{A K}^{-1}\left(\omega_{i}\right)=\frac{C_{V} T}{\rho v_{s}^{2}} \frac{\omega_{s}^{2} \tau_{T H}}{\left(1+\omega_{s}^{2} \tau_{T H}^{2}\right)} \gamma^{2}
$$

where $T$ is the temperature, $\rho$ the mass density, $v_{s}$ the group velocity of the mode $s$ and $\bar{\gamma}^{2}$ is the expected value of the square of the Grüneisen parameter taken over the entire spectrum of the thermal phonons. Due to the lack of direct experimental determination of $\bar{\gamma}$ and $\tau_{T H}$, the alignment of the damping models has relied on adjusting of these parameters to fit experimental data [23]. In fact, the only direct measurement available of $\bar{\gamma}$ is for modes a few high-symmetry points of the Brillouin zone [35]. The alternative is to infer $\bar{\gamma}$ from Density Functional Theory (DFT) calculations or using the second and third order elastic constants [36]. In the case of $\tau_{T H}$, a kink in the slope of the mechanical mode attenuation-frequency plot is used as indicative of the frequency cut-off between the intrinsic damping regimes from which $\tau_{T H}$ is extracted $[28,37,38]$. However, it has been shown that this slope depends on the direction of the phonon propagation [23]. In reference [25] we showed that the Akhieser model did not describe the experimental data of mechanical mode decay in Si ultrathin membranes, when a single value of $\tau_{T H}$ was used, regardless of the membranes thickness. As the real value of $\tau_{T H}$ is unclear, we used the constant value of $\tau_{T H}=17$ ps taken from reference [24], which provided the best fit to the acoustic attenuation in bulk silicon.

The thermal conductivity of thin layers and membranes, $\kappa_{\text {film }}$, decreases appreciably compared to the bulk counterpart, $\kappa_{b u l k}$. This reduction is mainly associated with the shortening of the phonon mean free path $\left(\Lambda_{m f p}\right)$ and concomitantly with the phonon lifetime $\left(\tau_{T H}\right)$, due to diffuse phonon scattering at the boundaries [19-22]. Introducing equation (1) in equation (2) the 
intrinsic phonon relaxation rate in the Akhieser regime can be rewritten as a function of the film thermal conductivity $\kappa_{\text {film }}$ :

$$
\tau_{A K}^{-1}=3 T \frac{C_{V}}{\rho}\left(\frac{\bar{v}}{v_{s}}\right)^{2} \frac{\omega_{s}^{2}\left(\kappa_{\text {film }} / C_{V}\right)}{\left(\bar{v}^{4}+\left(3 \omega_{s} \kappa_{\text {film }} / C_{V}\right)^{2}\right)} \bar{\gamma}^{2}
$$

or as a function of the thermal diffusivity $\alpha=\kappa_{\text {film }} / C_{V}$ :

$$
\tau_{A K}^{-1}=3 T C_{P}\left(\frac{\bar{v}}{v_{s}}\right)^{2} \frac{\alpha \omega_{s}^{2}}{\left(\bar{v}^{4}+\left(3 \alpha \omega_{s}\right)^{2}\right)} \bar{\gamma}^{2}
$$

where $C_{P}$ the specific heat capacity.

Following the approach by Ziman [39], the extrinsic processes are modelled using only the surface roughness scattering, $\tau_{\mathrm{B}}$. The relaxation rate takes the form:

$$
\tau_{B}^{-1}\left(\omega_{s}\right)=\frac{v_{s}}{d} \frac{1-p\left(\omega_{s}\right)}{1+p\left(\omega_{s}\right)}
$$

where $d$ is the thickness of the membrane, $v_{s}$ is the phonon group velocity of the mode and $p\left(\omega_{s}\right)=\exp \left(-4\left(\eta \omega_{s} / v_{s}\right)^{2}\right)$ is the fraction of phonons with frequency $\omega_{s}$ that undergo specular reflection at the boundaries characterised by a roughness $\eta$.

The total lifetime $\tau_{T}$ of the specific mechanical mode of frequency $\omega_{s}$ can be obtained by combining the intrinsic and extrinsic contributions using Matthiessen's rule:

$$
\tau_{\mathrm{T}}^{-1}\left(\omega_{s}\right)=\tau_{\text {ext }}^{-1}\left(\omega_{s}\right)+\tau_{\text {int }}^{-1}\left(\omega_{s}\right)
$$

\section{Results}

Figure $1 a$ shows the experimentally determined room-temperature thermal conductivity of Si membranes of thickness from $\sim 400 \mathrm{~nm}$ to $9 \mathrm{~nm}$ [40-42] which undergoes an appreciable decrease with decreasing membrane thickness. The thermal conductivity model using the Fuchs-Sondheimer approach $[43,44]$ is seen to be in good agreement with the measured values. The details and parameters used in the simulation can be found in the supplementary information of Reference [41]. 

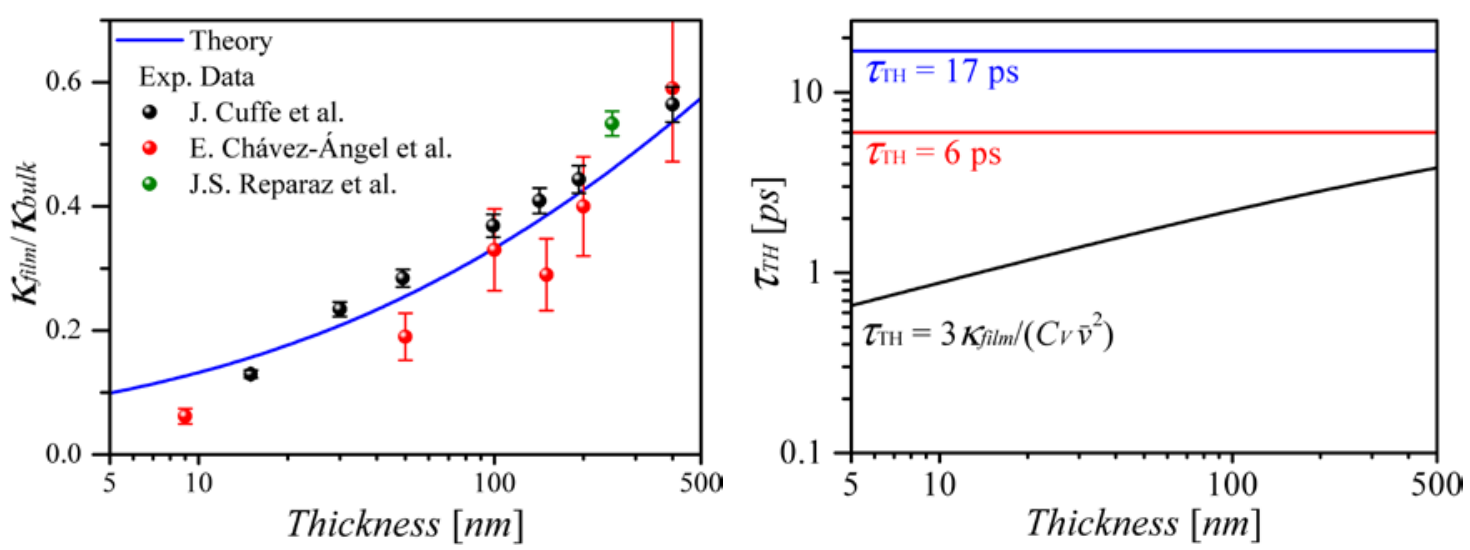

(a)

Figure 1 Thermal conductivity of Si membranes normalized to the bulk value, $\kappa_{\text {film }} / \kappa_{\text {bulk }}$, as function of membrane thickness. (a) The experimental data were obtained from thermal transient gradient (black dots) [40], single-laser Raman thermometry (red dots) [41] and two-laser Raman thermometry (green dot) [42], respectively. The theoretical description using the Fuchs-Sondheimer model is show by the solid blue line. (b) Calculated thermal phonons lifetime, $\tau_{T H}$, as a function of thickness (black line). Constant thermal phonons lifetime values of 17 and 6 ps are shown for comparison by blue and red lines, respectively.

An improved estimate of $\tau_{T H}$ can be obtained considering the thickness dependence of the thermal conductivity, $\kappa_{\text {film }}$, in the Equation (1). Figure $1 b$ shows the calculated $\tau_{T H}$ as a function of thickness together with the constant values $\tau_{T H}=17$ ps and 6 ps. While we took the first value ( $\tau_{T H}=17 \mathrm{ps)}$ from reference [24], where it was obtained as an adjustable parameter, the second value (6 ps) is directly derived from Equation (2) by taking $\kappa_{\text {film }}=\kappa_{\text {bulk }}=149 \mathrm{WK}^{-1} \mathrm{~m}^{-1}$ [45]. Then, once the dependence of $\tau_{T H}$ on membrane thickness has been determined, we introduce the total effect on the intrinsic phonon attenuation through equation (3).

Finally, we calculate the total lifetime $\tau_{T}$ of the silicon membranes considering the extrinsic surface-roughness boundary scattering $\tau_{B}$ and the intrinsic Akhieser damping effect $\tau_{A K}$. The values of $C_{V}, \bar{v}$, and $\rho$ used in these simulations were taken from the literature [35]. Since the Grüneisen parameter in silicon fluctuates between -1 and +1 depending on the direction and magnitude of the wavevector, it is possible to assume that the expected value of $\bar{\gamma}^{2}=\left\langle\gamma^{2}\right\rangle-$ $\langle\gamma\rangle^{2}$ is simply equal to 1 . Although this approximation fails at low temperatures where low frequency phonons with similar values of $\gamma$ are excited, at room temperature most acoustic phonons are excited and therefore the approach becomes reasonable [24]. Here we consider only 
the first dilatational mode of the membrane and in this case we can use the relation $\omega_{s}=\pi v_{s} / d$ to determine $v_{s}$. The value of $\eta=0.5 \mathrm{~nm}$ was taken from reference [25].

In figure 2 we compare the total relaxation time computed using the $\tau_{A K}$ discussed before and three the different values of the lifetime of the thermal phonons: two constant values ( $\tau_{T H}=17$ and 6 ps) and one thickness-dependent given by the equation (1). A better agreement with experimental data is obtained with the last thickness-dependent. As pointed out in reference [25], at frequencies above $100 \mathrm{GHz}$, equivalent to thickness $<50 \mathrm{~nm}$, extrinsic boundary scattering dominates the measured total phonon lifetime. In contrast, the intrinsic Akhieser damping becomes the dominant process at lower frequencies or thicknesses $>50 \mathrm{~nm}$.
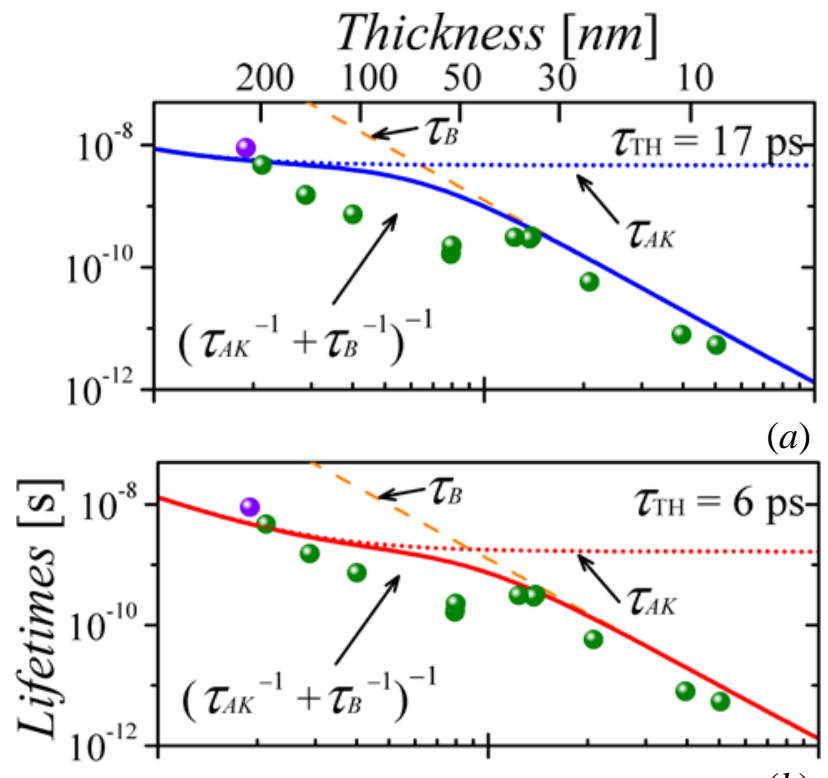

(b)

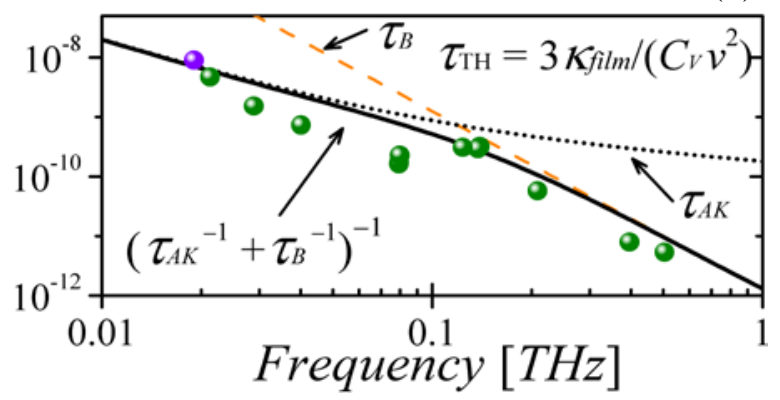

(c)

Figure 2 Experimental and theoretical phonon lifetime of the first-order dilatational mode in free-standing silicon membranes as a function of frequency. Data of free-standing Si membranes with thickness ranging from 194 to $8 \mathrm{~nm}$ (green dots) were taken from Reference [25] and the data point for a $222 \mathrm{~nm}$ thick membrane (violet dot) was taken from reference [30]. Solid lines are the calculated total contribution of each processes by summing the extrinsic boundary scattering, $\tau_{B}$ (dashed orange line) and the intrinsic Akhieser attenuation, $\tau_{A K}$, i.e., $\tau_{T}=$ $1 /\left(1 / \tau_{B}+1 / \tau_{A K}\right)$. Dotted blue (a) red (b) and black lines (c) are the intrinsic Akhieser attenuation dependence calculated for $\tau_{T H}=17 \mathrm{ps}$, (a) $\tau_{T H}=6 \mathrm{ps}$ (b) and $\tau_{T H}$ thickness-dependent (c), respectively. 
In our previous work [25] we simulated the intrinsic phonon attenuation at low frequencies using the Landau-Rumer model, however, we did not take into account the modification of the thermal phonon lifetime.

The modified thermal phonon lifetime has a direct consequence for the intrinsic upper limit of the $Q$-factor of nano-mechanical resonators, namely, a decrease in thermal conductivity can enhance or degrade the $Q$-factor depending on the frequency range. At low frequencies the $Q$ factor scales as $\sim 1 /\left(\omega \tau_{T H}\right)$ as is shown in Figure 3 and, consequently, smaller values of $\tau_{T H}$ result in higher $Q$-factor. However, at high frequencies, where the $Q$-factor scales as $\sim\left(\omega \tau_{T H}\right)$, decreasing $\tau_{T H}$ leads to the deterioration of the $Q$-factor. While a minimum value of the $Q$-factor is found at frequencies $f=1 / \tau_{T H}$, the lower the thermal conductivity the minima value is found at higher frequencies. In others words, in low thermal conductivity resonators, the $Q$-factor at high frequencies will be limited intrinsically by the poor heat dissipation.

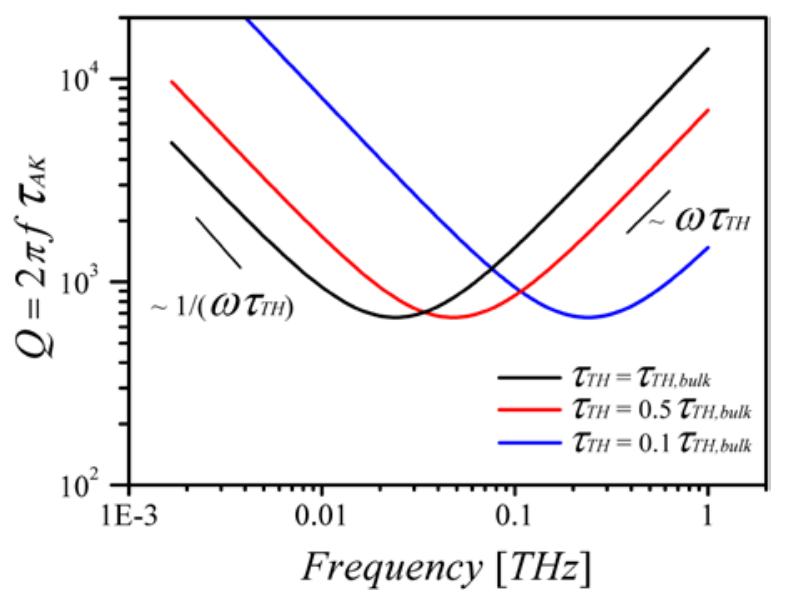

Figure 3 Frequency dependence of the Q-factor for different lifetime of the thermal phonon: silicon bulk values, $\tau_{T H, \text { bulk }}=6 \mathrm{ps}$, $50 \%$ of the bulk value, $\tau_{T H}=0.5 \tau_{T H, b u l k}=3$ ss and $10 \%$ of the bulk value, $\tau_{T H}=0.1 \tau_{T H, \text { bulk }}=0.6 \mathrm{ps}$.

In the example considered here, a Si membrane for which both phonon lifetimes and thermal conductivities were already known, the validation of the modified Akhieser model becomes easier. Moreover, the connection established in the model between the mechanical mode lifetime and thermal conductivity means that thermal transport parameters could be obtained from the mechanical mode lifetime or its $Q$-factor, only if the pure Akhieser mechanism is the dominant damping loss. This is particularly advantageous for contactless optical methods as pump-and-probe [25,26] or radio frequency spectroscopy [46] measurements since standard 
electrical methods to measure $\kappa$ require processing and calculation are not straightforward in complex shape nano/micro mechanical oscillators [47].

\section{Conclusions}

In this work we have revisited the intrinsic damping mechanism limiting the performance of mechanical resonators in view of the modification of thermal properties in nanostructures. This is exemplified in Si nanomembranes with mechanical mode frequencies above $10 \mathrm{GHz}$, where a reformulated Akhieser model is found to account very well for the measured decrease of the mechanical mode lifetime. We have demonstrated that for membranes it is not possible to assume a constant thermal phonon lifetime irrespectively of the membrane thickness, since modifications of the thermal conductivity due to surface scattering must be taken into account. We show that the reduction of the thermal conductivity in nano-resonators, and consequently a reduction of the thermal phonon lifetime, has a direct impact on the maximum $Q$-factor of a particular mechanical mode that depending on the frequency range could improve or degrade.

In addition, we suggest a method to extract thermal conductivity values from lifetime measurements, which opens the possibility to use the $Q$-factor as indicative of the thermal conductivity and/or diffusivity of nano-resonators.

\section{Acknowledgement}

The authors acknowledge the financial support from the FP7 project MERGING (grant nr. 309150), as well as the Spanish MINECO projects nanoTHERM (grant nr. CSD2010-0044) and TAPHOR (MAT2012-31392). E.C.A. gratefully acknowledges a Becas Chile 2010 CONICYT fellowship from the Chilean government.

\section{References}

[1] D. J. Young, C. A. Zorman, and M. Mehregany, in Springer Handbook Nanotechnology, edited by B. Bhushan (Springer Berlin Heidelberg, Berlin, Heidelberg, 2004), pp. 225-252. 
[2] Y. T. Yang, C. Callegari, X. L. Feng, K. L. Ekinci, and M. L. Roukes, Nano Lett. 6, 583 (2006).

[3] H. J. Mamin and D. Rugar, Appl. Phys. Lett. 79, 3358 (2001).

[4] A. N. Cleland and M. L. Roukes, Nature 392, 160 (1998).

[5] D. Rugar, R. Budakian, H. J. Mamin, and B. W. Chui, Nature 430, 329 (2004).

[6] T. J. Kippenberg and K. J. Vahala, Science 321, 1172 (2008).

[7] A. Schliesser, O. Arcizet, R. Rivière, G. Anetsberger, and T. J. Kippenberg, Nat. Phys. 5, 509 (2009).

[8] I. S. Grudinin, H. Lee, O. Painter, and K. J. Vahala, Phys. Rev. Lett. 104, 2 (2010).

[9] R. Rivière, S. Deléglise, S. Weis, E. Gavartin, O. Arcizet, A. Schliesser, and T. J. Kippenberg, Phys. Rev. A 83, 063835 (2011).

[10] V. Cimalla, J. Pezoldt, and O. Ambacher, J. Phys. D. Appl. Phys. 40, 6386 (2007).

[11] J. Weber, M. Link, R. Primig, D. Pitzer, W. Wersing, and M. Schreiter, IEEE Trans. Ultrason. Ferroelectr. Freq. Control 54, 405 (2007).

[12] J. Chan, T. P. M. Alegre, A. H. Safavi-Naeini, J. T. Hill, A. Krause, S. Gröblacher, M. Aspelmeyer, and O. Painter, Nature 478, 89 (2011).

[13] L. Ding, C. Baker, P. Senellart, A. Lemaitre, S. Ducci, G. Leo, and I. Favero, Appl. Phys. Lett. 98, 113108 (2011).

[14] X. Sun, X. Zhang, M. Poot, C. Xiong, and H. X. Tang, Appl. Phys. Lett. 101, 221116 (2012).

[15] A. H. Safavi-Naeini, J. T. Hill, S. Meenehan, J. Chan, S. Gröblacher, and O. Painter, Phys. Rev. Lett. 112, 153603 (2014).

[16] J. Gomis-Bresco, D. Navarro-Urrios, M. Oudich, S. El-Jallal, A. Griol, D. Puerto, E. Chavez, Y. Pennec, B. Djafari-Rouhani, F. Alzina, A. Martínez, and C. M. Sotomayor Torres, Nat. Commun. 5, 4452 (2014).

[17] A. Akhieser, J. Phys. USSR 1, 277 (1939).

[18] L. D. Landau and G. Rumer, Phys. Z. Sowjet. 11, 18 (1937).

[19] P. Hyldgaard and G. D. Mahan, in Thermal Conductivity 23, edited by K. E. Wilkes, R. B. Dinwiddie, and R. S. Graves (Technomics, 1996), pp. 172-182. 
[20] M. Asheghi, Y. K. Leung, S. S. Wong, and K. E. Goodson, Appl. Phys. Lett. 71, 1798 (1997).

[21] W. Liu and M. Asheghi, Appl. Phys. Lett. 84, 3819 (2004).

[22] G. H. Tang, Y. Zhao, G. X. Zhai, and C. Bi, J. Appl. Phys. 110, 046102 (2011).

[23] A. E. Duwel, J. Lozow, C. J. Fisher, T. Phillips, R. H. Olsson, and M. Weinberg, in Proc. SPIE 8031, Micro- Nanotechnol. Sensors, Syst. Appl. III, 80311C (May 13, 2011) (2011), p. 80311C-80311C-14.

[24] B. C. Daly, K. Kang, Y. Wang, and D. G. Cahill, Phys. Rev. B 80, 174112 (2009).

[25] J. Cuffe, O. Ristow, E. Chávez, A. Shchepetov, P.-O. Chapuis, F. Alzina, M. Hettich, M. Prunnila, J. Ahopelto, T. Dekorsy, and C. M. Sotomayor Torres, Phys. Rev. Lett. 110, 095503 (2013).

[26] A. A. Maznev, F. Hofmann, J. Cuffe, J. K. Eliason, and K. A. Nelson, Ultrasonics (2014).

[27] G. Rozas, M. F. P. Winter, B. Jusserand, A. Fainstein, B. Perrin, E. Semenova, and A. Lemaître, Phys. Rev. Lett. 102, 15502 (2009).

[28] A. A. Maznev, F. Hofmann, A. Jandl, K. Esfarjani, M. T. Bulsara, E. A. Fitzgerald, G. Chen, and K. A. Nelson, Appl. Phys. Lett. 102, 041901 (2013).

[29] A. Shchepetov, M. Prunnila, F. Alzina, L. Schneider, J. Cuffe, H. Jiang, E. I. Kauppinen, C. M. Sotomayor Torres, and J. Ahopelto, Appl. Phys. Lett. 102, 192108 (2013).

[30] A. Bruchhausen, R. Gebs, F. Hudert, D. Issenmann, G. Klatt, A. Bartels, O. Schecker, R. Waitz, A. Erbe, E. Scheer, J.-R. Huntzinger, A. Mlayah, and T. Dekorsy, Phys. Rev. Lett. 106, 77401 (2011).

[31] K. T. Regner, D. P. Sellan, Z. Su, C. H. Amon, A. J. H. McGaughey, and J. A. Malen, Nat. Commun. 4, 1640 (2013).

[32] J. P. Freedman, J. H. Leach, E. A. Preble, Z. Sitar, R. F. Davis, and J. A. Malen, Sci. Rep. 3, 2963 (2013).

[33] G. G. Sahasrabudhe and S. D. Lambade, J. Phys. Chem. Solids 60, 773 (1999).

[34] H. J. Maris, in Physical Acoustics: Principles and Methods, edited by W. P. Mason and R. N. Thurston (Academic Press, 1971), pp. 279-345.

[35] O. Madelung, U. Rössler, and M. Schulz, editors, Group IV Elements, IV-IV and III-V Compounds. Part a - Lattice Properties (Springer-Verlag, Berlin/Heidelberg, 2001). 
[36] G. P. Srivastava, The Physics of Phonons (Taylor \& Francis, 1990).

[37] J. Hasson and A. Many, Phys. Rev. Lett. 35, 792 (1975).

[38] T. Zhu, H. Maris, and J. Tauc, Phys. Rev. B 44, 4281 (1991).

[39] J. M. Ziman, Electrons and Phonons: The Theory of Transport Phenomena in Solids (Oxford University Press, USA, 1960).

[40] J. Cuffe, J. K. Eliason, A. A. Maznev, K. C. Collins, J. A. Johnson, A. Shchepetov, M. Prunnila, J. Ahopelto, C. M. S. Torres, G. Chen, and K. A. Nelson, arXiv:1408.6747 (2014).

[41] E. Chávez-Ángel, J. S. Reparaz, J. Gomis-Bresco, M. R. Wagner, J. Cuffe, B. Graczykowski, A. Shchepetov, H. Jiang, M. Prunnila, J. Ahopelto, F. Alzina, and C. M. Sotomayor Torres, APL Mater. 2, 012113 (2014).

[42] J. S. Reparaz, E. Chavez-Angel, M. R. Wagner, B. Graczykowski, J. GomisBresco, F. Alzina, and C. M. Sotomayor Torres, Rev. Sci. Instrum. 85, 034901 (2014).

[43] K. Fuchs, Math. Proc. Cambridge Philos. Soc. 34, 100 (1938).

[44] E. H. Sondheimer, Adv. Phys. 1, 1 (1952).

[45] C. Glassbrenner and G. Slack, Phys. Rev. 134, A1058 (1964).

[46] M. Eichenfield, J. Chan, R. M. Camacho, K. J. Vahala, and O. Painter, Nature 462, 78 (2009).

[47] A. M. Marconnet, T. Kodama, M. Asheghi, and K. E. Goodson, Nanoscale Microscale Thermophys. Eng. 16, 199 (2012). 